\title{
Analysis of the concordance of 20 immunohistochemical tissue markers in metastasectomy lesions in patients with metastatic renal cell carcinoma: $A$ retrospective study using tissue microarray
}

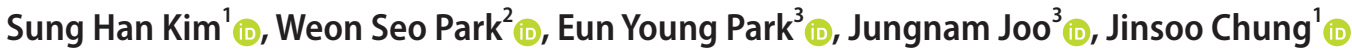 \\ 'Department of Urology, Center for Prostate Cancer, Research Institute and Hospital of National Cancer Center, Goyang, ${ }^{2}$ Department of Pathology, Center for Prostate \\ Cancer, Hospital of National Cancer Center, Goyang, ${ }^{3}$ Biostatistics Collaboration Team, Research Institute and Hospital of National Cancer Center, Goyang, Korea
}

Purpose: This study aimed to characterize the different expressions of 20 tissue markers in multiple metastatic lesions and organs in patients with metastatic renal cell carcinoma (mRCC).

Materials and Methods: Sixty-six patients with mRCC, harboring 162 metastasectomy tissue lesions (MTLs), were enrolled. Immunohistochemical analysis for the following tissue markers was performed: BAP1; CD31; CD 34; HIF1 $\alpha$ and 2 $\alpha$; Ki67; PS6; PBRM1; PDGFR $\alpha$ and $\beta$; PDL1; PSMA; PTEN; $\alpha$-SMA; TGase2; VEGFR1, 2, and 3; VHL loss; and CA9. Cases were identified pathologically using the semi-quantitative $\mathrm{H}$-score (0-300), including the intensity score $(0,1,2,3)$. The concordance rate was calculated as the number of patients with concordant binary score out of the total number of patients in that comparison.

Results: The specimens from 66 patients were divided into those from the same organs and those from different organs. Forty-two patients (44 cases) with 96 MTLs and 39 with 83 MTLs were examined. Among the 20 tissue markers, only BAP1, PSMA, VEGFR3, PDGFR $\alpha$, and pS6 tissue showed high concordance ratio $(>0.7)$ regardless of different metastatic tissues and different metastatic lesions within the tumor.

Conclusions: The study demonstrated the intratumoral heterogeneity of mRCC with a low-concordance index of most tissue markers. However, some had high concordance with a similar expression regardless of the metastatic organs, metastatic sites, or presence of recurrence.

Keywords: Carcinoma, renal cell; Immunohistochemistry; Neoplasm metastasis; Tissues 


\section{INTRODUCTION}

About one-third of newly diagnosed patients with renal cell carcinoma (RCC) have metastatic lesions, and another one-third of localized RCC progressed to recur and metastasize even after adequate surgical treatment [1,2]. For those with a known dismal prognosis of metastatic RCC (mRCC) due to chemotherapy, immunotherapy, or radiotherapy resistance, recent molecular genetic analyses led to a great advancement in improving survival outcomes in mRCC after introducing various new targeted and immunologic agents [3]. However, there still exist some impediments in achieving a remarkable progress in survival because of the side effects, drug resistance, diverse histology of RCC, intra- and intertumoral heterogeneity, and multiplicity of mRCC [4-6].

Various genetic and molecular approaches have been proposed to understand RCC histopathogenesis, including the von Hippel-Lindau-hypoxia-inducible factor-vascular endothelial growth factor (VHL-HIF-VEGF) pathway, phosphatidylinositol 3-kinase/protein kinase B/mammalian target of rapamycin (PI3K/Akt [activation of protein kinase]/ mTOR) pathway, and oncogenetic pathways of progression to metastasis, and of neovascularization in RCC $[3,7,8]$. However, the heterogeneity and different histology make it difficult to predict the treatment responses in $\mathrm{mRCC}$ in that some patients have both responsive primary and metastatic lesions, whereas the others demonstrate therapeutic responsiveness in either the primary or metastatic lesions [4,9-11].

One of the methods for understanding the molecular pathways and identifying the pathologic and morphologic characteristics of RCC is immunohistochemistry (IHC) staining of tissue microarrays (TMAs). It is a useful tool for understanding the roles of candidate genes in RCC pathogenesis, differentiating disease characteristics and expressive responses from primary and metastatic lesions, and determining the tissue expressions of tumor heterogeneity in different organ sites.

Therefore, in this study, 20 potential RCC-related tissue markers previously known as related to the oncogenesis of kidney cancer including vascular angiogenesis and to targeted therapy were applied immunohistochemically to the TMA of specimens from patients with $\mathrm{mRCC}$ who underwent metastatectomy to find out the intra-/interindividual as well as intratumoral heterogeneity of metastatic RCC lesions [3-11].
Table 1. Baseline characteristics $(n=66)$

\begin{tabular}{lc}
\hline \multicolumn{1}{c}{ Variable } & Value \\
\hline Age $(\mathrm{y})$ & $54.00 \pm 10.52$ \\
Body mass index $\left(\mathrm{kg} / \mathrm{cm}^{2}\right)$ & $24.41 \pm 2.71$ \\
Tumor size $(\mathrm{cm})$ & $7.27 \pm 3.09$ \\
Sex & $48(72.7)^{\mathrm{a}} / 18(27.3)^{\mathrm{b}}$ \\
American Society of Anesthesiologists & $15(26.8)^{\mathrm{a}} / 41(73.2)^{\mathrm{b}}$ \\
$\quad$ score $1 / 2+3($ miss $=10)$ & \\
Clinical stage T1-2/T3-4 or N+ (miss $=7)$ & $38(64.4)^{\mathrm{a}} / 21(35.6)^{\mathrm{b}}$ \\
Furhman grade $1+2 / 3+4($ miss=3) & $20(31.7)^{\mathrm{a}} / 43(68.3)^{\mathrm{b}}$ \\
Sarcomatoid differentiation (miss=4) & $57(91.9)^{\mathrm{a}} / 5(8.1)^{\mathrm{b}}$ \\
Necrosis (miss=4) & $37(59.7)^{\mathrm{a}} / 25(40.3)^{\mathrm{b}}$ \\
Lymphovascular invasion $($ miss $=4)$ & $49(79.0)^{\mathrm{a}} / 13(21.0)^{\mathrm{b}}$ \\
\hline
\end{tabular}

Values are presented as mean \pm standard deviation or number (\%). ${ }^{\mathrm{a}}:$ Male, ${ }^{\mathrm{b}}:$ female.

\section{MATERIALS AND METHODS}

\section{Patients' inclusion criteria and tissue samples}

Between 2001 and 2017, a total of 180 patients with mRCC were selected along with their 288 metastatic specimens. After a review of medical records, only 66 patients with mRCC, harboring 162 metastasectomy tissue lesions (MTLs), were enrolled in the study with the exclusion of patients without follow-up history and survival outcome. All samples were reviewed blindly, retrospectively and pathologically, by a uropathologist (WSP) with a 15-year experience assisted by a urologist (SHK) in accordance with the guidelines of World Health Organization/International Society of Urological Pathology (ISUP) consensus conference [12]. This study was approved by the Institutional Review Board of the National Cancer Center (approval number: NCC 20150219). The need for informed consent was waived by the board. The enrolled patients' medical records were obtained from a previously prospectively registered RCC registry database of the institution (Table 1).

\section{IHC and assessment of TMA}

TMA and IHC were performed in accordance with the previous studies using TMAs made by this group [13,14]. TMA blocks were built using representative tumor areas and paired normal control tissue from formalin-fixed, paraffin-embedded tumor material [15] and marked on standard hematoxylin/eosin (H\&E)-stained sections for the expressions of tissue markers.

The following 20 tissue biomarkers were immunohistochemically stained on the MTL TMA: BRCA1-associated protein-1 (ubiquitin carboxy-terminal hydrolase) (BAP1), polybromo 1 (PBRM1), phosphorylated S6 (pS6), phosphatase and tensin (PTEN), tissue glutaminase, protein-glutamine $\gamma$-glu- 
Table 2. Marker distribution tables with the sum score and positive/negative distribution within the same metastatic organs from the same patients with $\mathrm{mRCC}$

\begin{tabular}{|c|c|c|c|c|c|c|c|}
\hline Markers & $\mathrm{n}$ & Median & Min. & Max. & Negative & Positive & Concordance rate \\
\hline BAP1 loss & 96 & 280 & 10 & 360 & $95(99.0)$ & $1(1.0)$ & 0.977 \\
\hline PBRM1 loss & 96 & 150 & 0 & 290 & 77 (80.2) & 19 (19.8) & 0.659 \\
\hline PS6 & 96 & 0 & 0 & 280 & $84(87.5)$ & $12(12.5)$ & 0.818 \\
\hline PTEN loss & 96 & 270 & 0 & 300 & 79 (82.3) & $17(17.7)$ & 0.682 \\
\hline TGase2 & 96 & 225 & 0 & 290 & $47(49.0)$ & $49(51.0)$ & 0.568 \\
\hline PDL1 & 96 & 10 & 0 & 290 & $84(87.5)$ & $12(12.5)$ & 0.750 \\
\hline VHL loss & 48 & 10 & 0 & 300 & $13(27.1)$ & $35(72.9)$ & 0.583 \\
\hline HIF1 & 48 & 20 & 10 & 280 & 44 (91.7) & $4(8.3)$ & 0.750 \\
\hline HIF2 & 48 & 270 & 20 & 300 & $14(29.2)$ & $34(70.8)$ & 0.833 \\
\hline VEGFR1 & 48 & 165 & 10 & 290 & $25(52.1)$ & $23(47.9)$ & 0.583 \\
\hline VEGFR2 & 48 & 150 & 10 & 290 & $29(60.4)$ & $19(39.6)$ & 0.333 \\
\hline VEGFR3 & 48 & 20 & 10 & 280 & 44 (91.7) & $4(8.3)$ & 0.917 \\
\hline PDGFR $\alpha$ & 48 & 280 & 150 & 290 & $3(6.3)$ & $45(93.8)$ & 0.917 \\
\hline PDGFR $\beta$ & 48 & 150 & 10 & 290 & $29(60.4)$ & 19 (39.6) & 0.333 \\
\hline CA9 & 96 & 225 & 0 & 290 & 45 (46.9) & $51(53.1)$ & 0.500 \\
\hline CD31 & 48 & NA & NA & NA & $28(58.3)$ & $20(41.7)$ & 0.500 \\
\hline CD34 & 48 & NA & NA & NA & $27(56.2)$ & $21(43.8)$ & 0.333 \\
\hline SMA & 48 & 20 & 5 & 50 & $41(85.4)$ & $7(14.6)$ & 0.667 \\
\hline PSMA & 96 & 0 & 0 & 60 & $93(96.9)$ & $3(3.1)$ & 0.932 \\
\hline Ki67 & 96 & 0 & 0 & 30 & $50(52.1)$ & 46 (47.9) & 0.432 \\
\hline
\end{tabular}

Values are presented as number only or number (\%).

mRCC, metastatic renal cell carcinoma; Min., minimum; Max., maximum; NA, not available.

tamyltransferase (TGase-2), programmed cell death 1 ligand (PDL1), carbonic anhydrase 9 (CA9), prostate-specific membrane antigen (PSMA), Ki67, $\alpha_{-}$SMA, CD31, CD34, VEGR1, VEGFR2, VEGFR3, PDGFR $\alpha$, PDGFR $\beta$, HIF1 $\alpha$, HIF2 $\alpha$, and VHL tissue biomarkers.

The percentage of cells stained $(0 \%-100 \%)$ and the staining intensity were assessed within the nuclei and the cytoplasm of malignant cells and compared to that of the paired benign cells. The cases were identified pathologically by a senior uropathologist (WSP) blinded to the clinical outcome using the semi-quantitative $\mathrm{H}$-score (0-300), including the intensity score ( 0 for negative, $1+$ for weak, $2+$ for moderate, and $3+$ for strong). The intensity score was classified into two categories (negative or positive) to examine the concordance rate of the expressions of markers between different organs, and different sites of the same organ.

\section{Statistical analysis}

The immunostaining results for the 20 tissue biomarkers were analyzed under three conditions: between different sites of the same organ (Table 2), different organs (Table 3), and same organ with recurrent sites (Table 4). Only one tissue or tissues with additional surgery in less than 1 month were excluded from the analysis.
To examine the concordance rate of the 20 tissue markers, the $\mathrm{H}$-score or intensity score was converted into binary variables according to specific criteria in each marker. The concordance rate was calculated as the number of patients with concordant binary score out of the total number of patients in that comparison. All statistical analyses were performed using R project 3.3.3 and SAS 9.4 software (SAS Institute Inc,, Cary, NC, USA).

\section{RESULTS}

A total of 66 patients with 162 metastatic specimens obtained were analyzed to determine the expressing patterns of different tissue markers within tumors. The mean age and tumor size were 54 years and $7.27 \mathrm{~cm}$, respectively (Table 1). The patients' 162 specimens were divided into those from the same organs and those from different organs, including 80 from the kidneys, 36 from the lungs, 20 from the bone, 7 from the brain, 4 from the lymph nodes, and 15 from other organs. A total of 96 metastatic tumor lesions (MTL) from 42 patients (44 cases) and 83 MTLs from 39 patients were made TMAs to examine the concordance of the 20 immunohistochemical tissue markers within the same and different organs, respectively. The H-score or intensity score was 
Table 3. Marker distribution tables with the sum score and positive/negative distribution within different metastatic organs from the same patients with mRCC

\begin{tabular}{|c|c|c|c|c|c|c|c|}
\hline Markers & $n$ & Median & Min. & Max. & Negative & Positive & Concordance rate \\
\hline BAP1 loss & 83 & 250 & 10 & 300 & $80(96.4)$ & $3(3.6)$ & 0.923 \\
\hline PBRM1 loss & 83 & 50 & 0 & 290 & $64(77.1)$ & $19(22.9)$ & 0.667 \\
\hline PS6 & 83 & 10 & 0 & 280 & $73(88)$ & $10(12)$ & 0.744 \\
\hline PTEN loss & 83 & 270 & 0 & 300 & $73(88)$ & $10(12)$ & 0.795 \\
\hline TGase2 & 83 & 170 & 0 & 290 & $43(51.8)$ & $40(48.2)$ & 0.615 \\
\hline PDL1 & 83 & 10 & 0 & 290 & $67(80.7)$ & $16(19.3)$ & 0.718 \\
\hline VHL loss & 41 & 10 & 0 & 290 & $17(41.5)$ & $24(58.5)$ & 0.462 \\
\hline HIF1 & 41 & 20 & 10 & 270 & $38(92.7)$ & $3(7.3)$ & 0.923 \\
\hline HIF2 & 41 & 270 & 20 & 290 & $11(26.8)$ & $30(73.2)$ & 0.462 \\
\hline VEGFR1 & 41 & 150 & 10 & 290 & $26(63.4)$ & $15(36.6)$ & 0.385 \\
\hline VEGFR2 & 41 & 150 & 10 & 300 & $24(58.5)$ & $17(41.5)$ & 0.385 \\
\hline VEGFR3 & 41 & 20 & 10 & 280 & 39 (95.1) & $2(4.9)$ & 0.923 \\
\hline PDGFR $\alpha$ & 41 & 280 & 150 & 290 & $3(7.3)$ & $38(92.7)$ & 0.846 \\
\hline PDGFR $\beta$ & 41 & 150 & 10 & 280 & $24(58.5)$ & $17(41.5)$ & 0.462 \\
\hline CA9 & 83 & 200 & 0 & 290 & $40(48.2)$ & $43(51.8)$ & 0.410 \\
\hline CD31 & 41 & NA & NA & NA & $23(56.1)$ & 18 (43.9) & 0.615 \\
\hline CD34 & 41 & NA & NA & NA & $19(46.3)$ & $22(53.7)$ & 0.385 \\
\hline SMA & 41 & 10 & 5 & 50 & $38(92.7)$ & $3(7.3)$ & 0.846 \\
\hline PSMA & 83 & 0 & 0 & 60 & $82(98.8)$ & $1(1.2)$ & 0.974 \\
\hline Ki67 & 83 & 0 & 0 & 30 & $44(53)$ & $39(47)$ & 0.615 \\
\hline
\end{tabular}

Values are presented as number only or number (\%).

mRCC, metastatic renal cell carcinoma; Min., minimum; Max., maximum; NA, not available.

converted into binary variables to calculate the concordance rate. However, there was no gold standard to convert the $\mathrm{H}$ score to a binary variable, so it was classified follows: BAP1, PBRM1, PTEN, and VHL (H-score 10 vs. >10); PS6, TGase2, PDL1, HIF1, HIF2, VEGFR1, VEGFR2, VEGFR3, PDGFR $\alpha$, PDGFR $\beta$, and CA9 (H-score <200 vs. $\geq 200$ ); CD31 and CD34 (intensity score $0+1$ vs. $2+3$ ); SMA (H-score $\leq 40$ vs. $>40$ ); PSMA and Ki67 (H-score 0 vs. $>0$ ).

Within the 96 MTS of the same organs, the BAP1, PSMA, VEGFR3, PDGFR $\alpha$ ( $>0.9$ concordance rate), PS6, HIF2 ( $>0.8$ concordance rate), PDL1, and HIF1 ( $>0.7$ concordance rate) exhibited highly concordant expressions, even though they originated from different MTLs (Table 2 and Figs. 1-5).

As for the expression of different metastatic organs in the same 39 patients, BAP1, PSMA, HIF1, VEGFR3 ( $>0.9$ concordance rate), PDGFR $\alpha$, SMA ( $>0.8$ concordance rate), PS6, PDL1, and PTEN ( $>0.7$ concordance rate) showed consistent results, despite being obtained from different organs (Table 3 and Figs. 1-5).

For 26 patients who exhibited recurrence within the same organ, the tissue samples were re-stained for the markers, and MTLs were analyzed to examine the concordance of the recurrent site within the same organ. BAP1, PSMA $(>0.9$ concordance rate), PS6, VEGFR3, PDGFR $\alpha$ ( $>0.8$ concordance rate), PTEN, and HIF2 ( $>0.7$ concordance rate) demonstrated concordance (Table 4 and Figs. 1-5).

A final summary of concordance greater than 0.7 between the different tissues of different metastatic lesions among 20 tissue markers was documented in the Table 5 . BAP1 loss, PSMA, VEGFR3, PDGFR $\alpha$ and pS6 markers had a significantly high concordance regardless of different metastatic tumors within the same organs, different metastatic organs, and metachronous tumors of the same metastatic organs within the same patients.

\section{DISCUSSION}

One of the challenges in systematically treating $\mathrm{mRCC}$ is the presence of different genetic clones in different subpopulations of the same tumor, termed as intratumoral heterogeneity and multiplicity, resulting in different therapeutic responses and the development of therapeutic resistances to systemic therapies [11]. Although various new molecular targeting agents enabled patients to prolong overall survival more than that in the past era of cytokine therapy [16], the prognostic survival of patients with $\mathrm{mRCC}$ is still not enough, necessitating further genetic, molecular, and mor- 
Table 4. Marker distribution tables with the sum score and positive/negative distribution within metachronous tumor lesions of the same metastatic organs from the same patients with mRCC

\begin{tabular}{|c|c|c|c|c|c|c|c|}
\hline Markers & $\mathrm{n}$ & Median & Min. & Max. & Negative & Positive & Concordance rate \\
\hline BAP1 loss & 60 & 280 & 20 & 300 & $60(100.0)$ & NA (NA) & 1.000 \\
\hline PBRM1 loss & 60 & 135 & 0 & 290 & $51(85.0)$ & $9(15.0)$ & 0.679 \\
\hline PS6 & 60 & 10 & 0 & 280 & $51(85.0)$ & $9(15.0)$ & 0.821 \\
\hline PTEN loss & 60 & 275 & 0 & 300 & $50(83.3)$ & $10(16.7)$ & 0.714 \\
\hline TGase2 & 60 & 155 & 0 & 290 & $33(55.0)$ & $27(45.0)$ & 0.500 \\
\hline PDL1 & 60 & 10 & 0 & 290 & $50(83.3)$ & $10(16.7)$ & 0.679 \\
\hline VHL loss & 33 & 10 & 0 & 300 & $10(30.3)$ & $23(69.7)$ & 0.667 \\
\hline HIF1 & 33 & 20 & 10 & 280 & $30(90.9)$ & $3(9.1)$ & 0.667 \\
\hline HIF2 & 33 & 270 & 20 & 290 & $7(21.2)$ & $26(78.8)$ & 0.778 \\
\hline VEGFR1 & 33 & 180 & 10 & 290 & $17(51.5)$ & $16(48.5)$ & 0.667 \\
\hline VEGFR2 & 33 & 150 & 10 & 290 & $18(54.5)$ & $15(45.5)$ & 0.333 \\
\hline VEGFR3 & 33 & 20 & 10 & 280 & 30 (90.9) & $3(9.1)$ & 0.889 \\
\hline PDGFR $\alpha$ & 33 & 280 & 150 & 290 & $2(6.1)$ & 31 (93.9) & 0.889 \\
\hline PDGFR $\beta$ & 33 & 150 & 10 & 290 & 21 (63.6) & $12(36.4)$ & 0.333 \\
\hline CA9 & 60 & 205 & 0 & 280 & $28(46.7)$ & $32(53.3)$ & 0.607 \\
\hline CD31 & 33 & NA & NA & NA & $18(54.5)$ & 15 (45.5) & 0.556 \\
\hline CD34 & 33 & NA & NA & NA & 19 (57.6) & $14(42.4)$ & 0.333 \\
\hline SMA & 33 & 20 & 5 & 50 & $28(84.8)$ & 5 (15.2) & 0.667 \\
\hline PSMA & 60 & 0 & 0 & 60 & 59 (98.3) & $1(1.7)$ & 0.964 \\
\hline Ki67 & 60 & 0 & 0 & 30 & $32(53.3)$ & $28(46.7)$ & 0.464 \\
\hline
\end{tabular}

Values are presented as number only or number (\%).

mRCC, metastatic renal cell carcinoma; Min., minimum; Max., maximum; NA, not available.

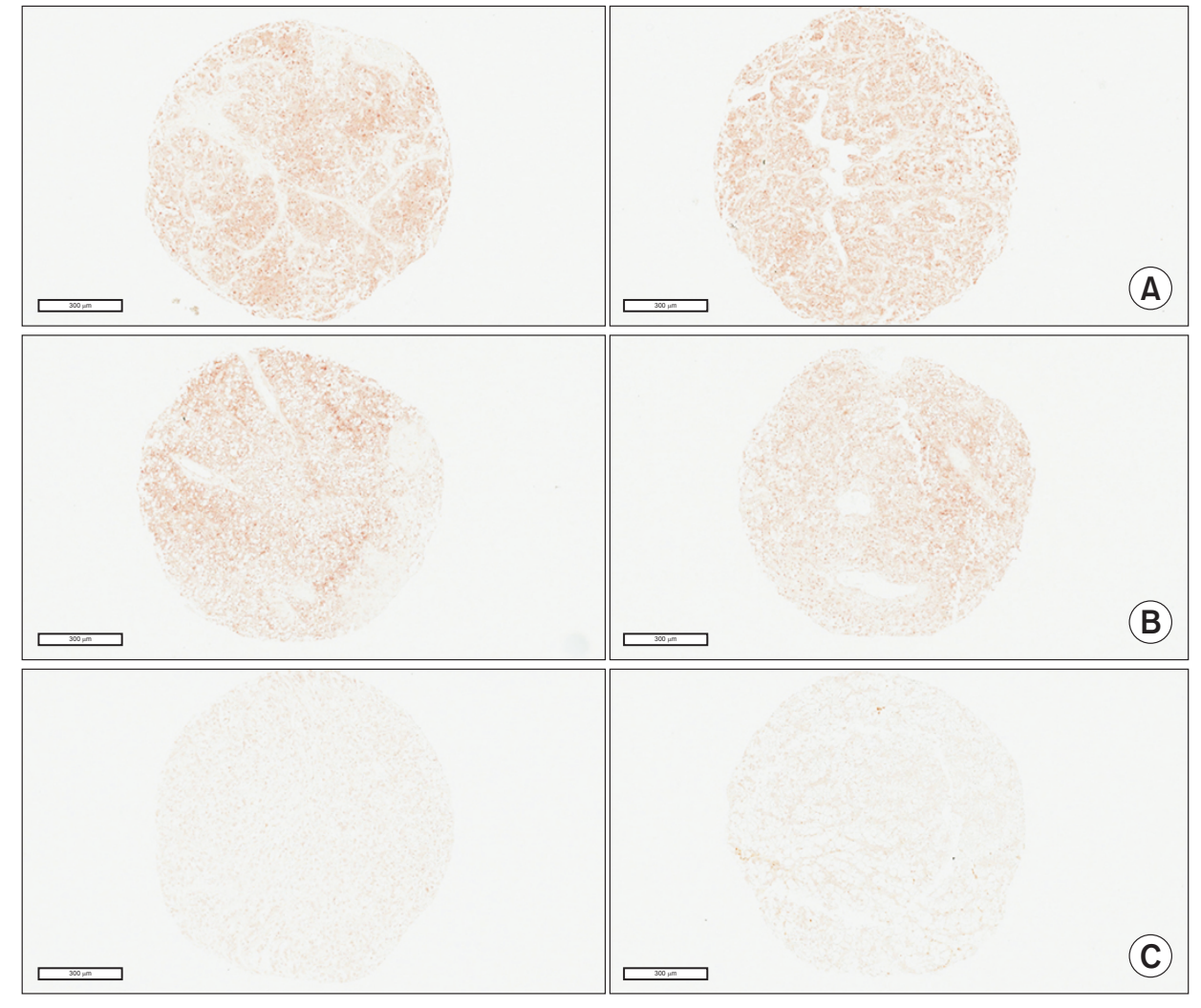

Fig. 1. One patient's immunohistochemistry of BAP1: (A) two separate metastatic tumors within the same lung, (B) two different metastatic tumors from two different metastatic organs such as lung and brain, (C) two metachronous tumors of same metastatic bone. Magnification: $\times 100$. 

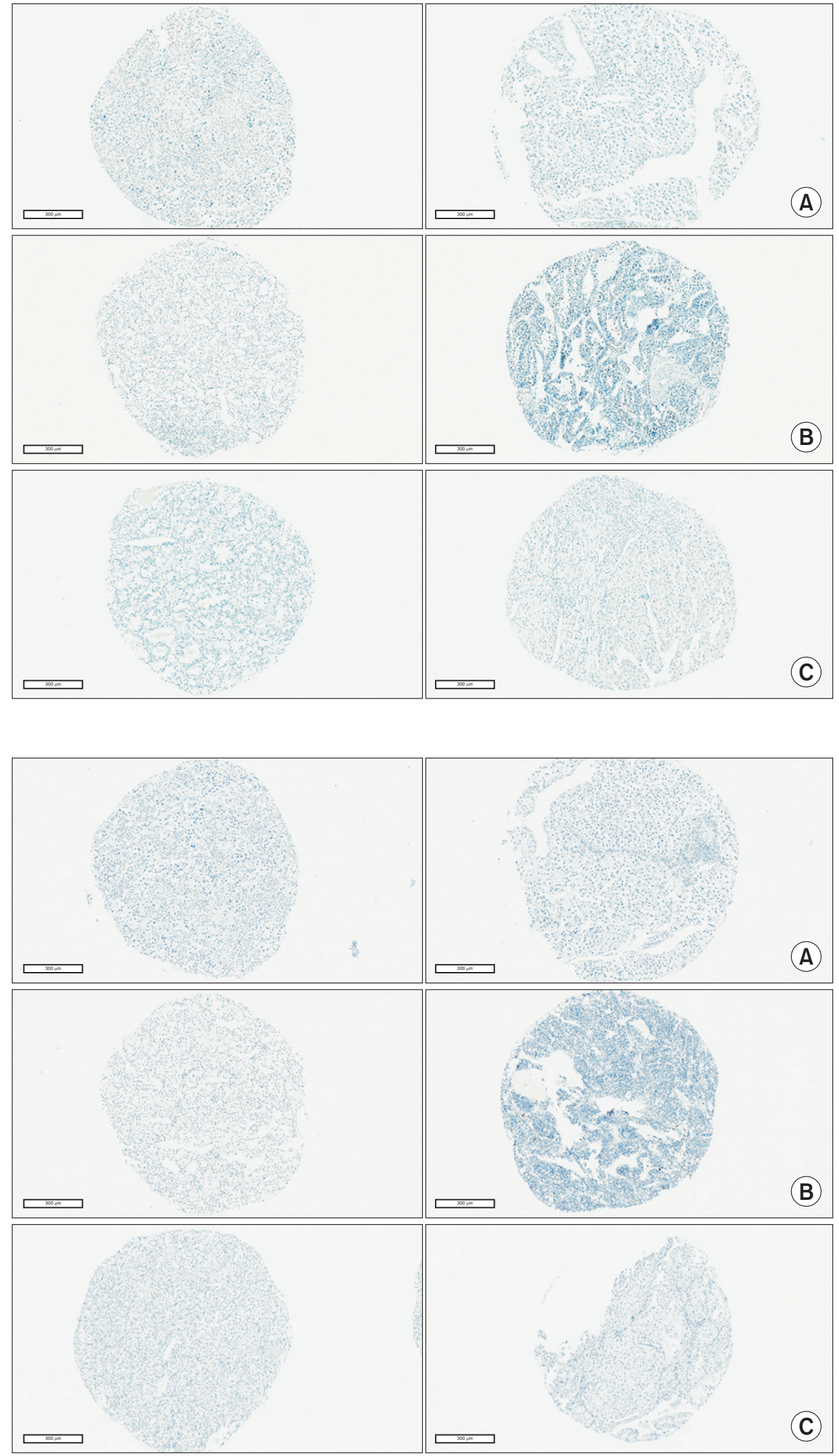

Fig. 2. One patient's immunohistochemistry of pS6: (A) two separate metastatic tumors within the same lung, (B) two different metastatic tumors from two different metastatic organs such as lung and femur, (C) two metachronous tumors of same metastatic lung. Magnification: $\times 100$.

Fig. 3. One patient's immunohistochemistry of PSMA: (A) two separate metastatic tumors within the same lung, (B) two different metastatic tumors from two different metastatic organs such as lung and femur, (C) two metachronous tumors of same metastatic organ. Magnification: $\times 100$.

phological researches for understanding mRCC.

The 20 tissue markers in this study were known to be significantly related to the VHL-HIF-VEGF pathway, PI3K/
Akt/mTOR pathway, and metastatic neovascularization of mRCC $[3,7,8]$. In order to define the intertumoral and intratumoral differences, the correlation of their expressions 

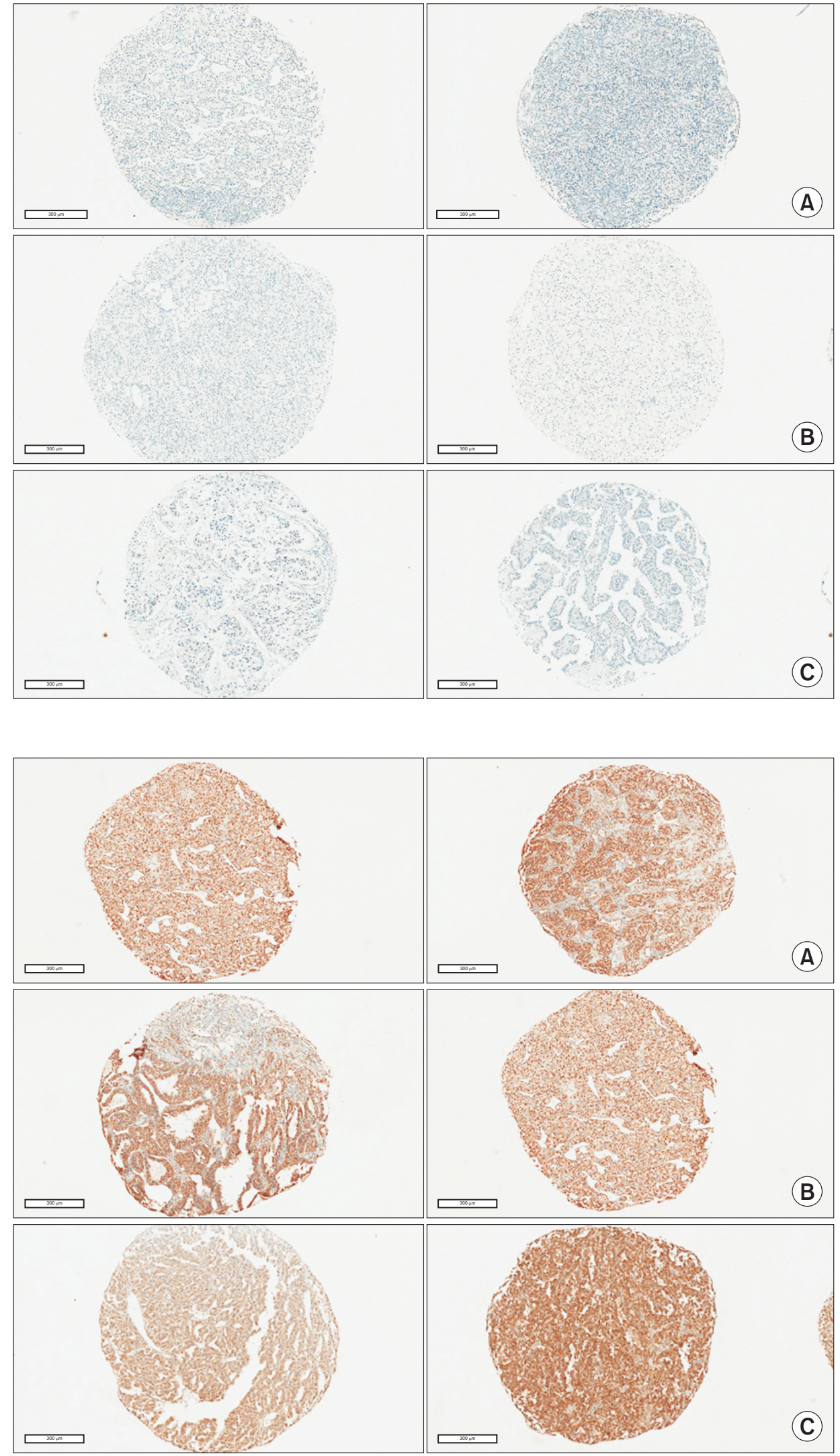

(C)
Fig. 4. One patient's immunohistochemistry of VEGFR3: (A) two separate metastatic tumors within the same lung, (B) two different metastatic tumors from two different metastatic organs such as lung and femur, (C) two metachronous tumors of same metastatic lung. Magnification: $\times 100$. were analyzed within tumors from the same intrapersonal organs and different organs, as well as between primary and recurrent tumors of the same sites within the same meta- static tumors using immunohistochemical analysis of TMA. Five tissue markers had high correlation not influenced by different metastatic organs, intrapersonal differences, and 
Table 5. Concordance score $>0.7$ for the mRCC markers among 20 tissue markers

\begin{tabular}{lll}
\hline \multicolumn{1}{c}{ A } & \multicolumn{1}{c}{ B } & \multicolumn{1}{c}{ C } \\
\hline BAP1loss $^{\mathrm{a}}$ & BAP1loss $^{\mathrm{a}}$ & BAP1loss $^{\mathrm{a}}$ \\
PSMA $^{\mathrm{a}}$ & PSMA $^{\mathrm{a}}$ & PSMA $^{\mathrm{a}}$ \\
VEGFR3 $^{\mathrm{a}}$ & VEGFR3 $^{\mathrm{a}}$ & VEGFR3 $^{\mathrm{a}}$ \\
PDGFRa $^{\mathrm{a}}$ & PDGFRa $^{\mathrm{a}}$ & PDGFRa $^{\mathrm{a}}$ \\
PS6 & PS6 $^{\mathrm{a}}$ & PS6 $^{\mathrm{a}}$ \\
PDL1 & PDL1 & \\
HIF1 & HIF1 $^{\mathrm{a}}$ & \\
HIF2 & & HIF2 \\
& PTENloss $^{\text {PTENloss }}$ \\
\hline
\end{tabular}

A different metastatic tumors within the same organs, $B$ different metastatic organs, and $C$ metachronous tumors of the same metastatic organs within the same patients.

${ }^{\mathrm{a}}$ :Represents concordance $\geq 0.8$.

metachronous timing of metastatic tumors within the same sites (BAP1 loss, PS6, VEGFR3, PDGFR $\alpha$, and PSMA, concordance rate $\geq 0.7$, Table 2 ). The other tissue markers were influenced by the aforementioned factors with completely different expressions within the same metastatic tumors, the same intrapersonal organs, and between recurrent and primary metastatic lesions.

This led to some information on the systemic therapeutic schema of life-threatening metastatic tumor complications of mRCC. The BAP1 loss, PS6, VEGFR3, PDGFR $\alpha$, and PSMA would be the best candidate targets controlling metastatic tumors in that the immunosuppressive effect of primary tumors was eliminated after primary tumor removal by cytoreductive nephrectomy, and the immunity defense system reactivates so as to concentrate on the metastatic tumors. With these five-specific tissue markers, the therapeutic responsiveness would be enhanced more efficiently in systemic targeted therapies on metastatic lesions, resulting in the improved survival of patients with $\mathrm{mRCC}$. In addition, the use of HIF1 and 2 and PDL1 inhibitors might be another additional excellent strategy for reducing the metastatic tumor complications in patients with synchronous mRCC, irrespective of different metastatic organs and interpersonal differences.

The aforementioned complications in genomic mutational and tumorigenic analyses were based on the intertumoral and intratumoral heterogeneity whereby the number of biopsied samples, metastatic sites, metastatic organs, and tumor microenvironment influenced the concordance of expressions in tumor tissues. For example, although there is no consensus as to how many samples should be taken during biopsies, larger numbers of biopsied specimens showed higher correlation [17]. Sankin et al. [18] biopsied 3 to 5 regions of tumors from 14 patients to examine for 5 specific mutations (VHL, PBRM1, BAP1, and the other two tissue markers) and demonstrated that a minimum of 3 samples are required to detect mutations with $90 \%$ certainty. Abel et al. [19] showed that quadruple biopsy demonstrated higher concordance with nephrectomy specimens in terms of tumor grade $(57.8 \%$ vs. $31.2 \%)$. This study used mostly 2 or 3 core biopsied specimens, and about 20\% were from 4 to 5 biopsied specimens. However, no studies have ever demonstrated the concordance of 20 tissue markers related to $\mathrm{mRCC}$ in 162 metastatic tissues from different lesions and organs from at least 30 patients with $\mathrm{mRCC}$.

An important consideration in planning systemic therapy for $\mathrm{mRCC}$ is the differences in primary and metastatic lesions. A logical approach to assess the differences in primary and metastatic lesions would be to analyze the expression of the genes and signaling pathways targeted by these molecules in the various tumor and metastatic lesions from each patient, similar to this study. This research team (Chung et al.) has continuously tried to identify the morphological expressions of known prognostic tissue markers of $\mathrm{mRCC}$ to determine the tumor characteristics of $\mathrm{mRCC}$, and to investigate the carcinogenetic mechanism of metastasis in RCC and the therapeutic resistance to systemic targeted therapy. Three previous immunohistochemical TMA studies [20,21], Abstract in J Clin Oncol 2019;37(7 Suppl):643) revealed no significant correlations of expressions among BAP1 loss, PBRM1 loss, pS6, PTEN loss, TGase2, PDL1, CA9, PSMA, and Ki67 between primary and metastatic lesions in patients with mRCC who underwent cytoreductive nephrectomy with metastatectomy [20]; pS6 and Ki67 were as significant factors for poor survival after adjusting for the significant prognostic clinicopathological parameters in patients with localized RCC who underwent radical nephrectomy [21]; and VHL1 loss, PBRM1 loss,TGAse-2, PDL1, VEGFR1, CD31, CD34, and pS6 were as significant prognostic markers in primary tumors of patients with $\mathrm{mRCC}$ who underwent cytoreductive nephrectomy (Abstract published in J Clin Oncol 2019;37(7 Suppl):643).

This study had several limitations, including its retrospective design with a small number of patient-matched samples and TMA specimens, intraindividual and interindividual interpretational errors, and some technical errors in TMA preparation. Most importantly, the small sample size and absence of gold standard for converting the H-score might influence the concordance indices of tissue markers with small deviating expressions within the TMA whereby the distribution of some of the highly concordant markers 
had shifted to one side. However, this was the first pathological concordance report of various tissue markers to prove the heterogeneity, multiplicity, and metachronous diversity of mRCC with intralesional and interlesional differences. Some markers had highly concordant expressions regardless of the different organs, lesions, and recurrent time intervals. Furthermore, this understanding of the different expressions of important tissue markers could provide some information not only on finding new targeting microenvironmental and tumorigenic markers but also approaching the progression and resistance mechanism after using systemic targeted therapies and immune checkpoint inhibitors $[3,8,22]$.

\section{CONCLUSIONS}

The findings of the study demonstrated the intratumoral heterogeneity of mRCC with the use of several tissue markers. Some markers had different expressions with a low-concordance index, whereas some had high concordance with a similar expression regardless of the metastatic organs, metastatic sites, or presence of recurrence.

\section{CONFLICTS OF INTEREST}

The authors have nothing to disclose.

\section{ACKNOWLEDGMENTS}

This study was supported by the Korean National Cancer Center Grants (no.1710290-1).

\section{AUTHORS' CONTRIBUTIONS}

Drafting of the manuscript: Sung Han Kim, Eun Young Park, and Jinsoo Chung. Critical revision of the manuscript: Sung Han Kim, Jungnam Joo, Weon Seo Park, Eun Young Park, and Jinsoo Chung. Obtaining funding: Jinsoo Chung. Administrative, technical, or material support: Weon Seo Park, Eun Young Park, and Jinsoo Chung. Supervision: Jungnam Joo, Weon Seo Park, Eun Young Park, and Sung Han Kim. Approval of the final manuscript: Jungnam Joo and Sung Han Kim.

\section{REFERENCES}

1. Motzer RJ. New perspectives on the treatment of metastatic renal cell carcinoma: an introduction and historical overview. Oncologist 2011;16 Suppl 2:1-3.

2. Ferlay J, Steliarova-Foucher E, Lortet-Tieulent J, Rosso S, Coe- bergh JW, Comber H, et al. Cancer incidence and mortality patterns in Europe: estimates for 40 countries in 2012. Eur J Cancer 2013;49:1374-403.

3. Jonasch E, Gao J, Rathmell WK. Renal cell carcinoma. BMJ 2014;349:g4797.

4. Beksac AT, Paulucci DJ, Blum KA, Yadav SS, Sfakianos JP, Badani KK. Heterogeneity in renal cell carcinoma. Urol Oncol 2017;35:507-15.

5. Moch H, Montironi R, Lopez-Beltran A, Cheng L, Mischo A. Oncotargets in different renal cancer subtypes. Curr Drug Targets 2015;16:125-35.

6. Tomaszewski JJ, Uzzo RG, Smaldone MC. Heterogeneity and renal mass biopsy: a review of its role and reliability. Cancer Biol Med 2014;11:162-72.

7. Liontos M, Trigka EA, Korkolopoulou P, Tzannis K, Lainakis $\mathrm{G}$, Koutsoukos K, et al. Expression and prognostic significance of VEGF and mTOR pathway proteins in metastatic renal cell carcinoma patients: a prognostic immunohistochemical profile for kidney cancer patients. World J Urol 2017;35:411-9.

8. Skolarikos AA, Papatsoris AG, Alivizatos G, Deliveliotis C. Molecular pathogenetics of renal cancer. Am J Nephrol 2006;26:218-31.

9. de Velasco G, Krajewski KM, Albiges L, Awad MM, Bellmunt J, Hodi FS, et al. Radiologic heterogeneity in responses to antiPD-1/PD-L1 therapy in metastatic renal cell carcinoma. Cancer Immunol Res 2016;4:12-7.

10. Hong B, Yang Y, Guo S, Duoerkun S, Deng X, Chen D, et al. Intra-tumour molecular heterogeneity of clear cell renal cell carcinoma reveals the diversity of the response to targeted therapies using patient-derived xenograft models. Oncotarget 2017;8:49839-50.

11. Sanfrancesco JM, Cheng L. Complexity of the genomic landscape of renal cell carcinoma: Implications for targeted therapy and precision immuno-oncology. Crit Rev Oncol Hematol 2017;119:23-8.

12. Moch $\mathrm{H}$. [The $\mathrm{WHO} / \mathrm{ISUP}$ grading system for renal carcinoma]. Pathologe 2016;37:355-60. German.

13. Kim SH, Kim SH, Joung JY, Lee GK, Hong EK, Kang KM, et al. Overexpression of ERG and wild-type PTEN are associated with favorable clinical prognosis and low biochemical recurrence in prostate cancer. PLoS One 2015;10:e0122498.

14. Park WS, Ryu J, Cho KH, Choi MK, Moon SH, Yun T, et al. Human papillomavirus in oropharyngeal squamous cell carcinomas in Korea: use of G1 cycle markers as new prognosticators. Head Neck 2012;34:1408-17.

15. Vogel UF, Bueltmann BD. Simple, inexpensive, and precise paraffin tissue microarrays constructed with a conventional microcompound table and a drill grinder. Am J Clin Pathol 2006;126:342-8. 
16. Wahlgren T, Harmenberg U, Sandström P, Lundstam S, Kowalski J, Jakobsson $\mathrm{M}$, et al. Treatment and overall survival in renal cell carcinoma: a Swedish population-based study (20002008). Br J Cancer 2013;108:1541-9.

17. Gerlinger M, Horswell S, Larkin J, Rowan AJ, Salm MP, Varela I, et al. Genomic architecture and evolution of clear cell renal cell carcinomas defined by multiregion sequencing. Nat Genet 2014;46:225-33.

18. Sankin A, Hakimi AA, Mikkilineni N, Ostrovnaya I, Silk MT, Liang Y, et al. The impact of genetic heterogeneity on biomarker development in kidney cancer assessed by multiregional sampling. Cancer Med 2014;3:1485-92.

19. Abel EJ, Heckman JE, Hinshaw L, Best S, Lubner M, Jarrard DF, et al. Multi-quadrant biopsy technique improves diagnostic ability in large heterogeneous renal masses. J Urol 2015;194:886-91.

20. Kim SH, Park WS, Park EY, Park B, Joo J, Joung JY, et al. The correlation of tissue-based biomarkers in primary and metastatic renal cell carcinoma lesions: a tissue microarray study. Korean J Urol Oncol 2016;14:152-8.

21. Kim SH, Park WS, Park EY, Park B, Joo J, Joung JY, et al. The prognostic value of BAP1, PBRM1, pS6, PTEN, TGase2, PDL1, CA9, PSMA, and Ki-67 tissue markers in localized renal cell carcinoma: a retrospective study of tissue microarrays using immunohistochemistry. PLoS One 2017;12:e0179610.

22. Semeniuk-Wojtaś A, Stec R, Szczylik C. Are primary renal cell carcinoma and metastases of renal cell carcinoma the same cancer? Urol Oncol 2016;34:215-20. 